\section{SAT0067 RADIOGRAPHIC DAMAGE AND DISABILITY IN PATIENTS WITH RA IN RELATION TO DISEASE DURATION IN THE ERA OF BIOLOGICS. LONG-TERM RESULTS FROM THE SCQM COHORT}

K.A. Heinimann ${ }^{1}$, J. von Kempis ${ }^{1}$, R. Sauter ${ }^{2}$, M. Schiff ${ }^{3}$, H. Schulze-Koops ${ }^{4}$, R. Mueller ${ }^{1} \cdot{ }^{1}$ Division of Rheumatology; ${ }^{2}$ Clinical Trials Unit, Kantonsspital St. Gallen, St. Gallen, Switzerland; ${ }^{3}$ University of Colorado, Denver, United States; ${ }^{4}$ Rheumaeinheit, Medizinische Klinik IV, Klinikum der Universität München, Munich, Germany

Background: Rheumatoid arthritis (RA) is an inflammatory disease with a chronic course over many years. Long-term observations in the late 90s have demonstrated that RA is associated with progressive articular destruction and increasing disability over the course of the disease. When biologic treatment was introduced, a lot of studies were able to show a favourable influence on disease activity, joint destruction, and disability. However, to our knowledge, no long-term studies have been conducted investigating the radiographic outcome and disability in this new era of treatment with biologics.

Objectives: To analyse patients with RA for radiographic damage, disability and disease activity dependent on disease duration.

Methods: We included all RA patients from the Swiss Clinical Quality Management in rheumatoid arthritis (SCQM) with at least one clinical visit and information on the disease duration available in the database. The primary endpoint was the association between the radiographic destruction, assessed by Ratingen erosion scores at the last clinical visit, and disease duration. This endpoint was investigated by a multiple negative binomial regression model which corrected for the effect of gender, age, rheumatoid factor (RF), ACPA and therapeutic status regarding the use of anti-TNF drugs and methotrexate (MTX), as possible confounding factors. Disease activity (DAS 28) and disability (HAQ DI) in dependence of disease duration served as secondary outcomes, analysed by using a multiple linear regression model with corrections for the same potential confounders as described for the primary endpoint.

Results: The original 52'753 records on 8'678 patients resulted in 6'526 evaluable observations for the Ratingen score. Disease duration ranged between less than 1 and more than 65 years, with a median disease duration of 8.3 years, and past or current use of anti-TNF drugs in $58.4 \%$ of patients. We found a significant association between disease duration and radiographic destruction with an average increase of Ratingen scores by $8.3 \%$ each year $(95 \% \mathrm{Cl}$ from $8.1 \%$ to $8.6 \%$ ). The most significant predictor for less radiographic destruction was a negative rheumatoid factor. During the first 5 years of the disease, clinical activity and disability decreased over time. While DAS 28-scores remained on a stable level in patients with a disease duration of more than 5 years (median DAS 28 of 2.8), HAQ-DI scores increased continuously by 0.018 for each additional year.

Conclusions: In this RA cohort, patients showed a continuous increase of articular destruction and physical disability in parallel to disease duration, despite treatment with biologics in the majority of patients. Even though in the era of biologics a satisfactory control of disease activity can be achieved in most patients, RA remains a progressive disease leading to joint destruction and physical disability.

Disclosure of Interest: None declared

DOI: 10.1136/annrheumdis-2016-eular.4176

\section{SAT0068 CIRCULATING MIR-126 IS A MARKER OF DISEASE ACTIVITY IN PATIENTS WITH EARLY RA BUT DOES NOT DIFFERENTIATE PATIENTS AT RISK OF DEVELOPING RA}

K. Prajzlerová, R. Jandová, V. Hrušková, P. Hánová, H. Mann, K. Pavelka, J. Vencovský, L. Šenolt, M. Filková. Institute of Rheumatology and Department of Rheumatology, 1st Faculty of Medicine, Charles University in Prague, Prague, Czech Republic

Background: We have previously shown differential expression of some circulating miRNAs in patients with early RA (ERA) and their potential as markers of disease activity and treatment response.

Objectives: We aimed to investigate differentially expressed circulating miRNAs in clinically suspect arthralgia patients with positive antibodies to citrullinated peptide antigens (ACPA) who are at high risk of developing RA and compared them to ERA and established RA.

Methods: The study included $20 \mathrm{ACPA}+$ arthralgia patients, 25 treatment naïve ERA patients who met the 2010 ACR/EULAR criteria (disease duration $<6$ months), 26 patients with highly active established RA prior anti-TNF treatment initiation and 24 healthy controls $(\mathrm{HC})$. Clinical disease activity assessments (DAS28, SDAl) were performed. Ultrasound of 28 small joints was performed in ACPA + arthralgia patients to evaluate subclinical synovitis. Total RNA from plasma was isolated using phenol-chloroform extraction. A comprehensive analysis of miRNAs was performed using TaqMan ${ }^{\circledR}$ Low Density Array (TLDA) in 5-6 samples in each group. The expression of miR-126 was further validated by single assays in remaining samples and normalized to an average of 2 spike-in C. elegans controls. dCt was used for relative quantification.

Results: Patients with arthralgia showed no clinical and ultrasound evidence of arthritis (grey scale and power Doppler for single joint $\leq 1$ ) Of these, all were ACPA+, CRP $7.4 \pm 19.2 \mathrm{mg} / \mathrm{l}$. Treatment naïve ERA patients had active disease (DAS28 5.7 \pm 1.3 , CRP $20.7 \pm 23.2 \mathrm{mg} / \mathrm{l}), 60 \%$ were ACPA+. Patients with established RA all had high disease activity (DAS28 6.6 \pm 0.9 , CRP 28.1 \pm 31.3 $\mathrm{mg} / \mathrm{l}), 73 \%$ were ACPA+, had failed DMARDs therapy and were due to commence anti-TNF treatment. Out of the 380 miRNAs analysed by TLDA, 128 miRNAs were detected in HC, 129 in arthralgia, 44 in ERA and 25 in RA patients. TLDA analysis revealed $1.7 x$ higher levels of miR-126 in ACPA+ arthralgia patients compared to HC, and 27x and 63x lower levels in ERA and RA compared to HC, respectively. These data were suggestive of significant dysregulation of miR-126 in patients with active polyarthritis compared to ACPA+ arthralgia. However, further validation showed no difference in miR-126 levels among HC, arthralgia and ERA patients but the levels were 2.6x lower in RA patients compared to HC $(p=0.0037)$. The levels of miR-126 significantly negatively correlated with DAS28 $(r=-0.460 ; p=0.047)$, SDAl $(r=-0.535 ; p=0.018)$, SJC $(r=-0.504 ; p=0.028)$, and TJC $(r=-0.517 ; p=0.023)$ in treatment naïve ERA patients, but not in those with established disease. There was no correlation with CRP in any patient group. Conclusions: Higher miR-126 levels reflect lower disease activity in treatment naïve ERA patients. We hypothesize that significant downregulation of miR-126 levels in RA patients may be modified by previous exposure to multiple treatments. Acknowledgement: MHCR 023728 project

Disclosure of Interest: None declared

DOI: 10.1136/annrheumdis-2016-eular.4172 